\title{
4-Carboxymethoxy-5,7-Dinitroindolinyl-Glu: An Improved Caged Glutamate for Expeditious Ultraviolet and Two-Photon Photolysis in Brain Slices
}

\author{
Graham C. R. Ellis-Davies, ${ }^{1}$ Masanori Matsuzaki, ${ }^{2,3}$ Martin Paukert, ${ }^{4}$ Haruo Kasai, ${ }^{2}$ and Dwight E. Bergles ${ }^{4}$ \\ ${ }^{1}$ Department of Pharmacology and Physiology, Drexel University College of Medicine, Philadelphia, Pennsylvania 19102, ${ }^{2}$ Division of Biophysics, Center for \\ Disease Biology and Integrative Medicine, Faculty of Medicine, The University of Tokyo, Tokyo 113-0033, Japan, ${ }^{3}$ Precursory Research for Embryonic \\ Science and Technology, Japan Science and Technology Agency, Saitama, Japan, and ${ }^{4}$ The Solomon H. Snyder Department of Neuroscience, Johns Hopkins \\ School of Medicine, Baltimore, Maryland 21205-2105
}

Caged neurotransmitters are useful photochemical tools for selective stimulation of synapses and other transmitter receptors. Before illumination, the caged compound is biologically inert. Photolysis breaks a covalent bond, liberating the caged neurotransmitter. Release can be rapid, so the resultant synaptic stimulation can mimic a natural one (Matsuzaki et al., 2001). Uncaging does not replace traditional electrode stimulation; rather, it is a useful complement to it for several reasons: (1) a single transmitter is normally photoreleased, (2) stimulation of voltage-gated ion channels is not required for transmitter release, (3) receptors at many synapses can be activated simultaneously according to the area (or volume) of illumination, (4) unnatural amino acids can be photoreleased, and (5) subquantal or supraquantal neurotransmitter release is feasible.

Photochemical uncaging of neuro-

Received April 4, 2007; revised May 11, 2007; accepted May 13, 2007.

This work was supported by National Institutes of Health Grants GM65473 (G.C.R.E.-D. and H.K.), MH0717505 and NDS44564 (G.C.R.E.-D.), and PAR-02-059 and NS44261 (D.E.B.); Grants-in-Aid from the Ministry of Education, Culture, Sports, Science, and Technology of Japan (H.K. and M.M.); the Sumitomo Foundation (M.M.); the Human Frontier Science Program (RPG0071/2002 to G.C.R.E.-D. and H.K.); and the Center for NanoBio Integration, the University of Tokyo (H.K. and M.M.).

Correspondence should be addressed to Graham C. R. Ellis-Davies, Department of Pharmacology and Physiology, Drexel University College of Medicine, Philadelphia, PA 19102. E-mail: ged@drexel.edu.

DOI:10.1523/JNEUROSCI.1519-07.2007

Copyright $\odot 2007$ Society for Neuroscience $\quad$ 0270-6474/07/276601-04\$15.00/0 transmitters is especially useful when studying neurons in acutely isolated brain slices (Callaway and Yuste, 2002). Such intact tissue is the preparation of choice for many neuroscientists because it preserves much of the three-dimensional architecture of cellular connectivity that exists in vivo, yet, being ex vivo, permits relatively easy access for multielectrode stimulation and recording, along with pharmacological intervention. However, the very complexity of the brain slice preparation can restrict the reductionistic scientific drive to control and understand smaller structures such as single synapses. For example, each hippocampal CA1 neuron has $\sim 10,000$ synapses, so that the stimulation of a single synapse by traditional electrical means is at best haphazard or virtually impossible. Uncaging provides an answer to this quandary (Judkewitz et al., 2006). Because light can be focused and directed to any point across a neuron, it can be used to uncage known amounts of neurotransmitter at visually designated positions for defined periods, permitting controlled receptor activation of single synapses (Matsuzaki et al., 2001). Thus, the photolysis beam is a "magic wand" for synaptic physiology. Patterned light, however, is only half the answer; caged transmitters (e.g., caged glutamate) must be synthesized to fulfill the promise of the photostimulation method.
Caged neurotransmitters must satisfy several stringent chemical and biological design criteria to be useful for experimental neuroscience. From a biological point of view, the essential property of a caged transmitter is that it must be inert. From a chemical point of view, the photolabile molecule must release the biologically active substrate much faster than the process under study. AMPA-type glutamate receptors open with rise-times of $\sim 100-500 \mu \mathrm{s}$, so, ideally, the rate of glutamate uncaging should be $100,000 / \mathrm{s}$. Furthermore, the caged substrate must be released with decent photochemical efficiency. Two physicochemical properties define such efficiency: the extinction coefficient $(\varepsilon)$ and the quantum yield $(\phi)$. This study deals with the latter property. We have synthesized a new caged glutamate (4-carboxymethoxy-5,7-dinitroindolinylglutamate or CDNI-Glu) (Fig. 1) that releases glutamate with a $\phi=$ 0.5. This is approximately four to five times more efficient than the widely used 4-methoxy-7-nitroindolinylglutamate (MNI-Glu). We found that comparative uncaging with near-UV irradiation (350 $\mathrm{nm})$ or two-photon excitation $(720 \mathrm{~nm})$ of CDNI-Glu and MNI-Glu on the same neurons in cortical or hippocampal brain slices elicited postsynaptic currents consistent with their $\phi$. The increased photosensitivity of CDNI-Glu permitted quantal two-photon uncaging with a very short 
pixel dwell time (10 $\mu \mathrm{s})$ at photon fluxes well below the threshold for photodamage. Because uncaging of glutamate from CDNI-Glu is significantly more efficient than MNI-Glu, it may enable new types of photostimulation experiments in intact brain tissue.

\section{Synthesis and photochemistry}

The scheme for the synthesis of CDNIGlu is shown in Figure 1 (for details, see supplemental material, available at www.jneurosci.org). Two related routes to CDNI-Glu were developed, both starting from 4-hydroxyindole (Fig. 1, compound 1). Dinitration of the aromatic ring was the key step in the synthesis and proved to be troublesome. We found that the most chemically efficient way to do this was by sequential introduction of each nitro functionality. Thus, acid 6 (Fig. 1) was dissolved in trifluoroacetic acid (TFA), and $\mathrm{NaNO}_{3}$ was added as a solid; mononitration was essentially instantaneous. The products, compound 7 and 4-carboxymethoxy-7nitro-indolinyl (CNI)-Glu (Fig. 1), were separated by HPLC with $80 \%$ overall yield. The pure 5-nitro isomer (Fig. 1, compound 7) was then redissolved in TFA with $20 \mathrm{~mol}$ equivalents of $\mathrm{NaNO}_{3}$. The nitration reaction went to completion in $\sim 72 \mathrm{~h}$. CDNIGlu was isolated by HPLC with $25-28 \%$ yield from the 5-nitro compound (Fig. 1, compound 7).

Irradiation of solutions CDNI-Glu and MNI-Glu at $\mathrm{pH} 7.4$ showed that the quantum yield of photolysis of CDNI-Glu was $\sim 0.5$, six times higher than MNI-Glu. Nuclear magnetic resonance (NMR) analysis of the reaction mixture revealed that L-glutamate was released essentially quantitatively from CDNI-Glu (Fig. 1, box). Furthermore, NMR analysis of a photolysis solution of compound 7 (Fig. 1), CNIGlu, and CDNI-Glu showed that during the time required for complete photolysis of CDNI-Glu only $\sim 10 \%$ of the CNI-Glu was photolyzed (comparative irradiation of MNI-Glu and CNI-Glu revealed that the quantum yield of photolysis of the latter was $\sim 0.1$, data not shown.) These data imply that the quantum yield for release of L-glutamate from CDNI-Glu was $\sim 0.5$.

\section{CDNI-Glu uncaging on acutely isolated} brain slices

We compared the relative sizes of postsynaptic currents evoked by photolysis of CDNI-Glu and MNI-Glu on pyramidal neurons in acutely isolated cortical or hippocampal brain slices. The significantly higher quantum yield of glutamate uncaging from CNDI-Glu compared with
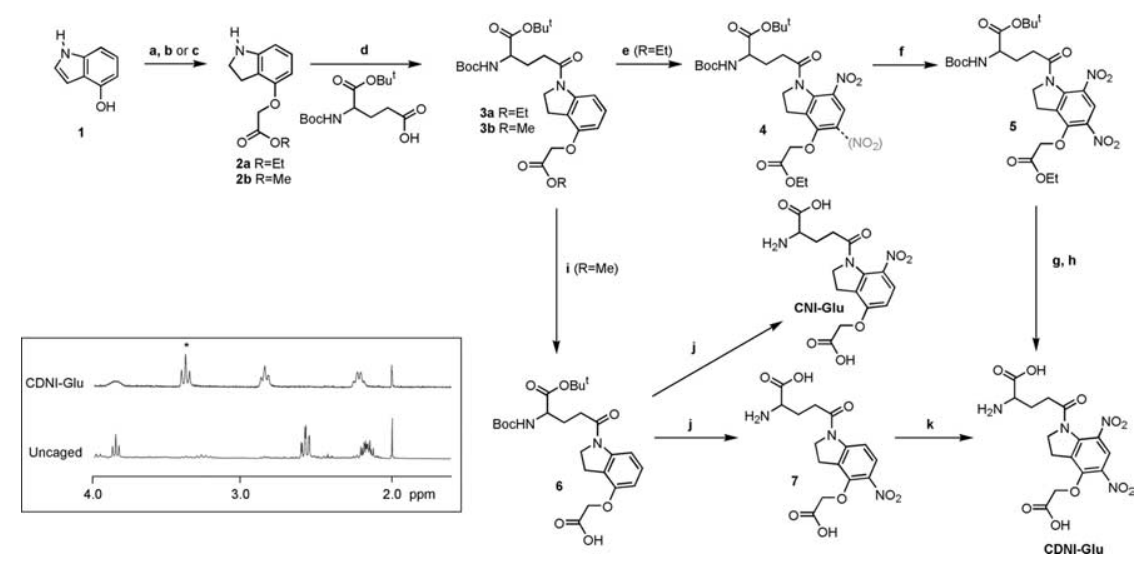

Figure 1. Synthesis of $\mathrm{CDNI}-\mathrm{Glu}$. The reagents and conditions are listed as follows: (a) ethylbromoacetate, $\mathrm{K}_{2} \mathrm{CO}_{3}$, acetone, room temperature $\left(\mathrm{RT}=23-25^{\circ} \mathrm{C}\right), 18 \mathrm{~h} ;(\boldsymbol{b}) \mathrm{NaBH}_{3} \mathrm{CN}, \mathrm{AcOH} ;(\boldsymbol{c})$ as Papageorgiou et al., 2004; (d) dicyclohexylcarbodiimide ( $\mathrm{R}=$ ethyl) or 1-(3-dimethylaminopropyl)-3-ethycarbodimide $\left(\mathrm{R}=\right.$ methyl); (e) claycop, acetic anhydride, $\mathrm{CCl}_{4}, \mathrm{RT}^{\prime}(\boldsymbol{f}) \mathrm{HNO}_{3}$, acetic anhydride, RT; (g) LiOH, THF, RT; (h) TFA, RT; (i) NaOH, MeOH, RT; (j) NaNO ${ }_{3}(1.2$ equ), TFA, RT, then HPLC isolation of 5 -isomer; ( $\boldsymbol{k}) \mathrm{NaNO}_{3}$ (20 equ), TFA, RT. Full details of the synthesis are in the supplemental material (available at www. jneurosci.org). The inset box shows NMRs in $\mathrm{D}_{2} \mathrm{O}$ of the amino acid portion of (DNI-Glu before and after photolysis: the caging chromophore is photolyzed (peak marked with * disappears), and glutamate is cleaning released.
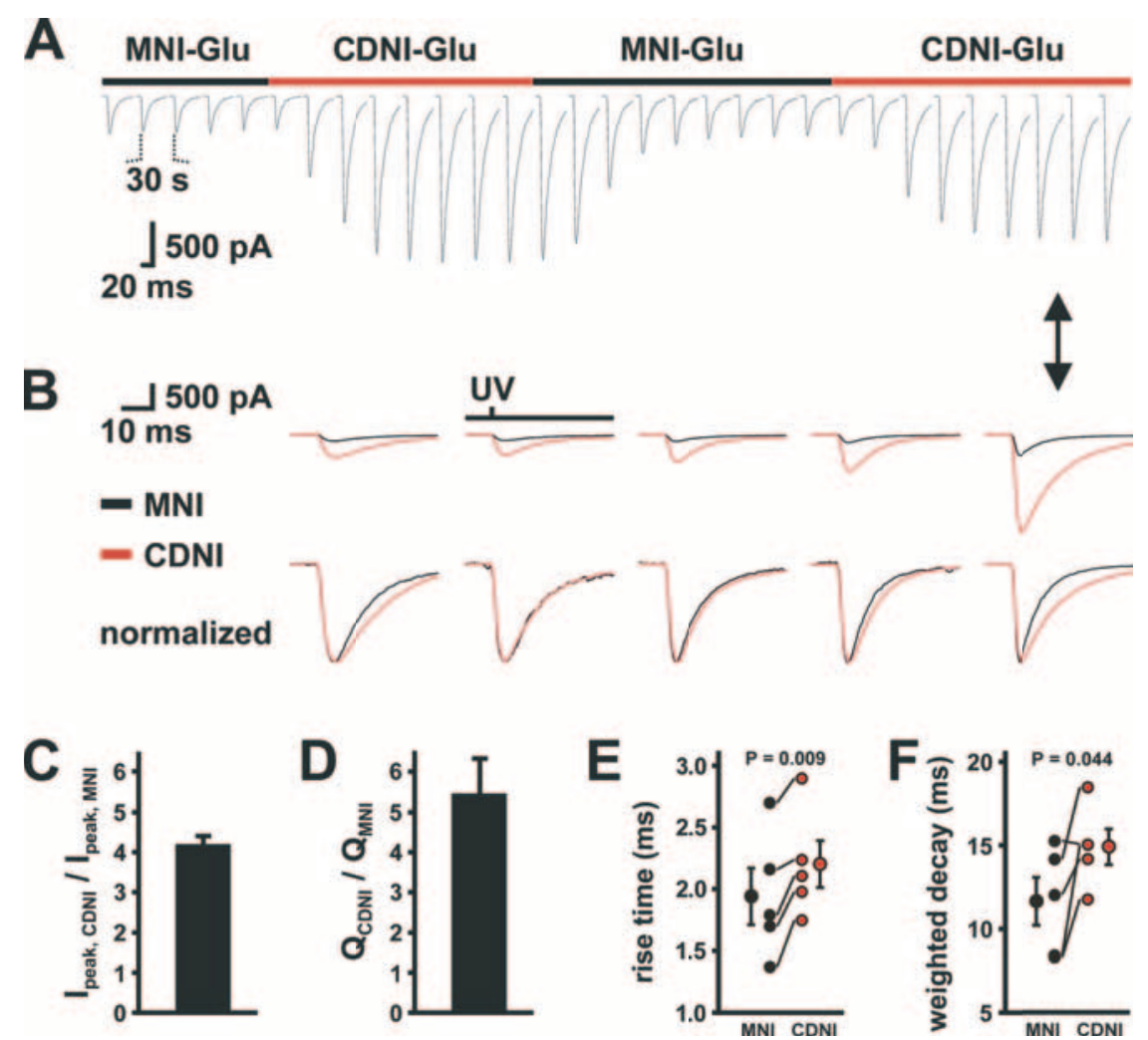

Figure 2. Comparative uncaging of CDNI-Glu and MNI-Glu on pyramidal neurons in acutely isolated cortical brain slices using UV irradiation. $\boldsymbol{A}$, The caged glutamates (concentration of $0.2 \mathrm{~mm}$ ) were locally perfused onto a pyramidal neuron through a wide bore application pipette. The photolysis beam $\left(333.6-363.8 \mathrm{~nm}\right.$; diameter, $\left.50 \mu \mathrm{m} ; 30 \mathrm{~mJ} / \mathrm{cm}^{2}\right)$ was focused through a $40 \times$ water objective lens (for full details, see Huang et al., 2005). B, Superimposed average traces comparing responses to photolysis of CDNI-Glu (in red) and MNI-Glu (in black) from five cells (top traces). The traces taken from the recording in $A$ are highlighted by the arrow. Normalization of the response to MNI-Glu to the peak amplitude of the response to CDNI-Glu uncaging (bottom traces) is shown. $C-\boldsymbol{F}$, Comparison of the peak currents, total charge, rise times, and decay times of the evoked currents from uncaging CNDI-Glu and MNI-Glu (the lines in $\boldsymbol{E}$ and $\boldsymbol{F}$ connect responses collected from individual cells). $p$ values were determined using the Student's paired $t$ test.

MNI-Glu enabled us to evoke larger currents with the same laser energy or use lower energies and cage concentrations, yet produce similar currents. The relative sizes of these currents were consistent with the photophysical properties of the two caged glutamates. For example, sequential near-UV photolysis of alternat- 
A
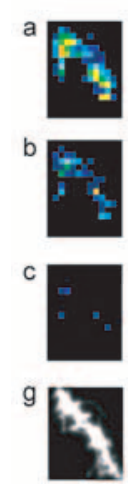

B
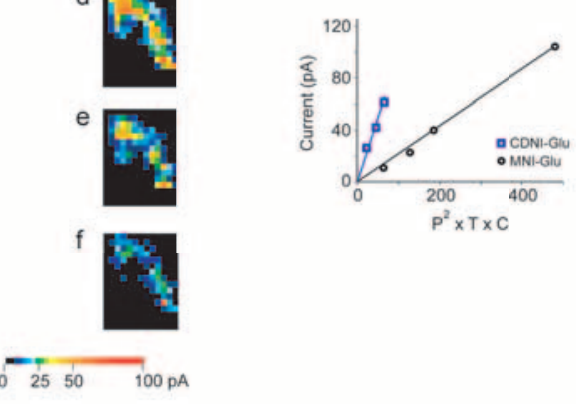

C

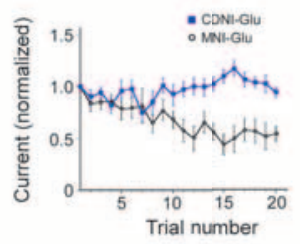

D

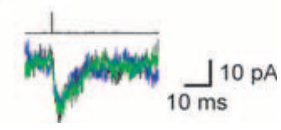

Figure 3. Comparative uncaging of CDNI-Glu and MNI-Glu on acutely isolated hippocampal brain slices using two-photon excitation. $A$, The caged glutamates ([CDNI-Glu $]=3.5 \mathrm{~mm}$; $[\mathrm{MNI}-\mathrm{Glu}]=10 \mathrm{mM})$ were locally perfused onto the same hippocampal CA1 neuron through an application pipette. A small dendritic region $(\boldsymbol{A g})$ was selected for two-photon mapping according to our standard procedure (Matsuzaki et al., 2001). The region of interest was divided into $13 \times 16$ pixels (size $0.71 \mu \mathrm{m}$ ). The photolysis beam (mode-locked Ti:sapphire laser tuned to $720 \mathrm{~nm}$ ) was focused through a $60 \times$ water objective lens (NA 0.9 ). Uncaging of MNI-Glu ( $\boldsymbol{A} \boldsymbol{a}-\boldsymbol{c})$ and CDNI-Glu ( $\boldsymbol{A d}-\boldsymbol{f})$ at three different energies $(\boldsymbol{a}, \boldsymbol{d}, 3.9 \mathrm{~mW}$ for $1.2 \mathrm{~ms} ; \boldsymbol{b}, \boldsymbol{e}, 3.9 \mathrm{~mW}$ for $0.8 \mathrm{~ms}$; $\boldsymbol{c}, \boldsymbol{f}, 2.7 \mathrm{~mW}$ for $0.8 \mathrm{~ms}$ ) produced different mapping intensities. Currents are displayed according to the pseudo-color scale. $\boldsymbol{B}$, Quantitative comparison of the photoevoked currents in $A$ with power $(P=3.9$ or $2.7 \mathrm{~mW})$, shutter time ( $\mathrm{T}=1.2$ or $0.8 \mathrm{~ms})$, and cage concentration (C) showed that CDNI-Glu (blue line) was 4.2 times more photosensitive than MNI-Glu (black line). C, Repetitive two-photon uncaging of CNDI-Glu (7 mM) or MNI-Glu ( $12 \mathrm{mM})$ at single spine heads using a short shutter open time (10 $\mu \mathrm{s}$ ) and high laser intensities [CNDI-Glu, $22 \mathrm{~mW}$ (blue) and MNI-Glu, $48 \mathrm{~mW}$ (black)]. Energy per flash for CDNI-Glu was approximately the same as in $\boldsymbol{A f}(n=9 ; \pm$ SEM). $\boldsymbol{D}$, Three examples of current traces induced by photolysis of CNDI-Glu (from $\boldsymbol{C}$ ). Black, blue, and green traces correspond to the first, 10th and 20th trials, respectively. The top trace showed the time of the illumination.

ing solutions of the same concentration of MNI-Glu and CDNI-Glu on the same cortical pyramidal neuron produced AMPA-receptor currents that were four to five times larger for CDNI-Glu than MNI-Glu (Fig. 2A-D). Under these conditions, the currents produced by uncaging CDNI-Glu had slightly slower rise and decay times than the currents produced by uncaging MNI-Glu $(n=5)$ (Fig. $2 E, F)$, presumably reflecting the prolonged activation of AMPA receptors as more glutamate was liberated; a larger quantity of glutamate would remain elevated for a longer period of time and activate AMPA receptors at a greater distance from the site of photoliberation. Application of CDNI-Glu $(200 \mu \mathrm{M}) \mathrm{did}$ not produce a change in holding current $(n=5)$ before UV exposure, indicating that the compound remains effectively caged in aqueous solution. Furthermore, the rapid kinetics of the currents produced by photolysis of CDNI-Glu are comparable with those described for MNI-Glu (Matsuzaki et al., 2001; Carter and Sabatini, 2004; Huang et al., 2005; Shoham et al., 2005; Sobczyk et al., 2005; Araya et al., 2006; Beique et al., 2006; Gasparini and Magee, 2006), suggesting that this new caged compound also does not act as a partial antagonist of AMPA receptors
MNI-Glu is the only caged neurotransmitter that has been reported to undergo diffraction-limited, two-photon photolysis (for review, see Judkewitz et al., 2006). Because of this, it has been used to stimulate synaptic events at single spine heads in acutely isolated and cultured brain slices (Matsuzaki et al., 2001; Carter and Sabatini, 2004; Sobczyk et al., 2005; Araya et al., 2006; Beique et al., 2006; Gasparini and Magee, 2006). Therefore, we compared the AMPA-receptor currents evoked by two-photon photolysis of CDNI-Glu and MNI-Glu. Using the mapping protocol we previously developed with MNI-Glu (Matsuzaki et al., 2001), we found that two-photon photolysis of solutions of either CDNI-Glu (3.5 mM) or MNI-Glu (10 mM) on the same dendritic arbor of a CA1 hippocampal neuron evoked robust currents (Fig. $3 A$ ). The currents from CDNI-Glu were approximately four to five times larger than those of MNI-Glu (Fig. 3B). This functional mapping was performed with a large pixel size $(0.7 \mu \mathrm{m})$, low powers $(2.7-3.9 \mathrm{~mW})$, and long shutter times $(0.8-1.2 \mathrm{~ms})$. We then reduced the pixel dwell time to $10 \mu \mathrm{s}$ and increased the laser power so the total two-photon efficiency during each uncaging event was similar (i.e., $2-5 \mu \mathrm{J}$ ) to that used in Figure $3 A$. Instead of multisite mapping, we focused the laser to isolated individual spine heads and tested how the evoked current stability varied with the number of uncaging pulses. We found that quantal uncaging of MNI-Glu (12 $\mathrm{mM})$ required large laser power $(48 \mathrm{~mW})$. This high peak power proved to be somewhat phototoxic after 10-20 uncaging events (Fig. $3 C$, black line). In contrast, CDNI-Glu (7 mM) required significantly less power $(22 \mathrm{~mW})$, and little or no phototoxicity was observed (Fig. 3C, blue line).

The raison d'être of neurons is to produce action potentials. The dendritic tree uses multiple (virtually) simultaneous synaptic inputs to produce such output. Because the amplitude of individual synaptic events is subthreshold for action potential generation, integration across synapses is a necessary prerequisite for neuronal function. One dream of synaptic physiologists is the elucidation of the rules for such synaptic integration. Recently, progress in this area has been made using two-photon uncaging of MNI-Glu (Gasparini and Magee, 2006). However, current microscope and chemical technology restricts the use of two-photon uncaging from faithfully mimicking simultaneous, multisite input across a dendritic arbor that must occur in normal brain function. This is because the speed of the currently deployed galvanometer drivers imposes a lower limit on the point-topoint movement time of $\sim 0.1 \mathrm{~ms}$ (Gasparini and Magee, 2006). Use of MNI-Glu in such experiments required an uncaging pixel dwell time of an additional $0.2 \mathrm{~ms}$, necessitating that each synaptic event occurs with a $0.3 \mathrm{~ms}$ spacing. With the introduction of CDNI-Glu, we have reduced the required pixel dwell time by a factor of 20. We hope this improvement will be a spur to the construction of two-photon uncaging microscopes with ultra-rapid beam steering capabilities. Combining such technology with CDNI-Glu will significantly compress the period for multisite uncaging and bring the "dream" closer to fulfillment.

\section{References}

Araya R, Jiang J, Eisenthal KB, Yuste R (2006) The spine neck filters membrane potentials. Proc Natl Acad Sci USA 103:17961-17966.

Beique J-C, Lin D-T, Kang M-G, Aizawa H, Takamiya K, Huganir RL (2006) Synapse-specific regulation of AMPA receptor function by PSD95. Proc Natl Acad Sci USA 103:19535-19540.

Callaway EM, Yuste R (2002) Stimulating neurons with light. Curr Opin Neurobiol 12:587-592. 
Carter AG, Sabatini BL (2004) State-dependent calcium signaling in dendritic spines of striatal medium spiny neurons. Neuron 44:483-493.

Gasparini S, Magee JC (2006) State-dependent dendritic computation in hippocampal CA1 pyramidal neurons. J Neurosci 26:2068-2100.

Huang YH, Sinha SR, Fedoryak OD, EllisDavies GC, Bergles DE (2005) Synthesis and characterization of 4-methoxy-7nitroindolinyl-D-aspartate, a caged compound for selective activation of glutamate transporters and $\mathrm{N}$-methyl-D-aspartate receptors in brain tissue. Biochemistry 44:3316-3326.

Judkewitz B, Roth A, Hausser M (2006) Dendritic enlightenment: using patterned twophoton uncaging to reveal the secrets of the brain's smallest dendrites. Neuron 50:180-183.

Matsuzaki M, Ellis-Davies GC, Nemoto T, Miyashita Y, Ino M, Kasai H (2001) Dendritic spine geometry is critical for AMPA receptor expression in hippocampal CA1 pyramidal neurons. Nat Neurosci 4:1086-1092.
Papageorgiou G, Lukeman M, Wan P, Corrie JE (2004) An antenna triplet sensitizer for 1-acyl-7-nitroindolines improves the efficiency of carboxylic acid photorelease. Photochem Photobiol Sci 3:366-373.

Shoham S, O'Connor DH, Sarkisov DV, Wang SS-H (2005) Rapid neurotransmitter uncaging in spatially defined patterns. Nat Methods 2:837-843.

Sobczyk A, Scheuss V, Svoboda K (2005) NMDA receptor subunit-dependent $\left[\mathrm{Ca}^{2+}\right]$ signaling in individual hippocampal dendritic spines. J Neurosci 25:6037-6046. 